\title{
Modelling constraints point to the ancient formation of the Moon
}

\author{
MAXWELL MARZBAN THIEMENS ${ }^{1,2}$, JONAS TUSCH ${ }^{3}$, \\ RAÚL O.C. FONSECA ${ }^{4}$, FELIPE P. LEITZKE ${ }^{5}$, MARIO \\ FISCHER-GÖDDE ${ }^{6}$, VINCIANE DEBAILLE ${ }^{7}$, PETER \\ SPRUNG $^{8}$ AND CARSTEN MÜNKER ${ }^{6}$ \\ ${ }^{1}$ Vrije Universiteit Brussel \\ ${ }^{2}$ Université Libre de Bruxelles \\ ${ }^{3}$ University of Cologne \\ ${ }^{4}$ Ruhr-University Bochum \\ ${ }^{5}$ Universidade Federal do Rio Grande do Sul \\ ${ }^{6}$ Universität zu Köln \\ ${ }^{7}$ Université Libre de Bruxelles. \\ ${ }^{8}$ Paul Scherrer Institut \\ Presenting Author: maxwellmt@gmail.com
}

The age and formation of the Moon remains a highly debated topic. Constraining this event can be achieved through first order observations (direct sample measurements) or modelling. However, differing variables used in numerical models can yield vastly different timescales for lunar formation. Here, we will synthesize relevant observations of the early Earth-Moon system to assess the robustness of different modelling constraints used in the lunar community. Starting from observations of the EarthMoon system, including the extreme chemical similarities between the Earth and Moon, we will examine the elemental and isotopic mass balance of tungsten (W), and correlate this with constraints from highly siderophile elements. To complement this, we will examine short-lived isotope systematics of Archean terrestrial rocks to provide first order constraints on the composition of the proto-Earth. Coupled ${ }^{182} \mathrm{~W}-{ }^{142} \mathrm{Nd}$ variations have been found in several Archean sample suites, providing evidence for early terrestrial silicate differentiation. If not taken into consideration, this can strongly bias conclusions on the lateaccretion history of the Earth-Moon system.

Novel isotope systems can always provide additional insights. In this regard, coupled ruthenium $(\mathrm{Ru}), \mathrm{W}$, and highly siderophile element (HSE) data can lend additional help to discriminate between the effects of late accretion and early silicate differentiation. The $\mathrm{Ru}$ isotope compositions of Greenland samples provide definite constraints for late-veneer contributions to the early Earth, showing that the Eoarchean mantle received at least $60 \%$ of the late veneer we observe in the present day mantle. This estimate provides further support for an important role of early silicate differentiation, that must have contributed to the $\mathrm{W}$ isotopic composition of Archean rocks. The lunar $\mathrm{W}$ isotope signature, meanwhile, is best explained as the result of the formation of a small lunar core.

The age of the Moon obtained through the Hf-W system is largely unaffected by the amount of ${ }^{182} \mathrm{~W}$ contributions suggested by $\mathrm{Ru}$ isotopes and HSE. Even assuming massive late veneer contributions beyond what HSE and Ru data suggest were feasible, the age of the Moon would shift less than 10 million years. As such, the Moon remains incontrovertibly old. 